\title{
Chandra Observations of the Antennae Galaxies (NGC 4038/39)
}

\author{
G. Fabbiano \\ Harvard-Smithsonian Center for Astrophysics, 60 Garden St., \\ Cambridge MA 02138, USA
}

\begin{abstract}
A deep ( 70ks) Chandra ACIS pointing at the merging system NGC 4038/39 gives us an exceptional detailed view of its X-ray emission and demonstrates the capabilities of Chandra's sub-arcsecond resolution. We detect an extraordinarily luminous population of pointlike sources, that may suggest $10-100 M_{\odot}$ Black Hole counterparts. We also detect a luminous hot ISM, with features ranging to large scale outflows to hot superbubbles.
\end{abstract}

\section{Introduction}

This is a preliminary report of the deep Chandra ACIS observation of the Antennae galaxies. This system was observed in December 1999 as part of the garanteed time observer program of Dr. S. S. Murray. These results and their interpretation for the global X-ray emission properties of this pair of merging galaxies are reported in Fabbiano, Zezas \& Murray (2000). Detailed studies of the discrete X-ray source population and of the hot and multi-phase ISM will follow in Zezas et al (2000, in preparation) and Fabbiano et al (2001, in preparation) respectively. Some of the material in this talk was also presented at the COSPAR symposium in Warsaw (Poland) in the summer 2000, and at the X-ray Astronomy 2000 conference in Palermo (Italy).

NGC 4038/39 are the nearest pair of colliding galaxies involved in a major merger $\left(D=29 \mathrm{Mpc}\right.$, for $\left.H_{0}=50\right)$. The merging interaction has caused widespread intense star formation in both galaxies, as evidenced by the rings of giant H II regions and bright stellar knots with luminosities up to $M_{V} \sim-16$ (Rubin et al 1970), which are resolved with the Hubble Space Telescope into typically about a dozen young star clusters (Whitmore \& Schweizer 1995).

Starburst galaxies can be spectacular X-ray emitters, since the enhanced star formation activity results in enhanced X-ray emission and in hot gaseous plumes and galaxian superwinds in the most extreme cases (e.g. Fabbiano 1988). This behaviour was also suggested by earlier X-ray studies of NGC 4038/39, although the distance of this system and the limited resolution and sensitivity of past X-ray observatories could not really let us 'see' what was going on (Fabbiano \& Trinchieri 1983; Read et al 1995; Sansom et al 1996; Fabbiano, Schweizer \& Mackie 1997). 


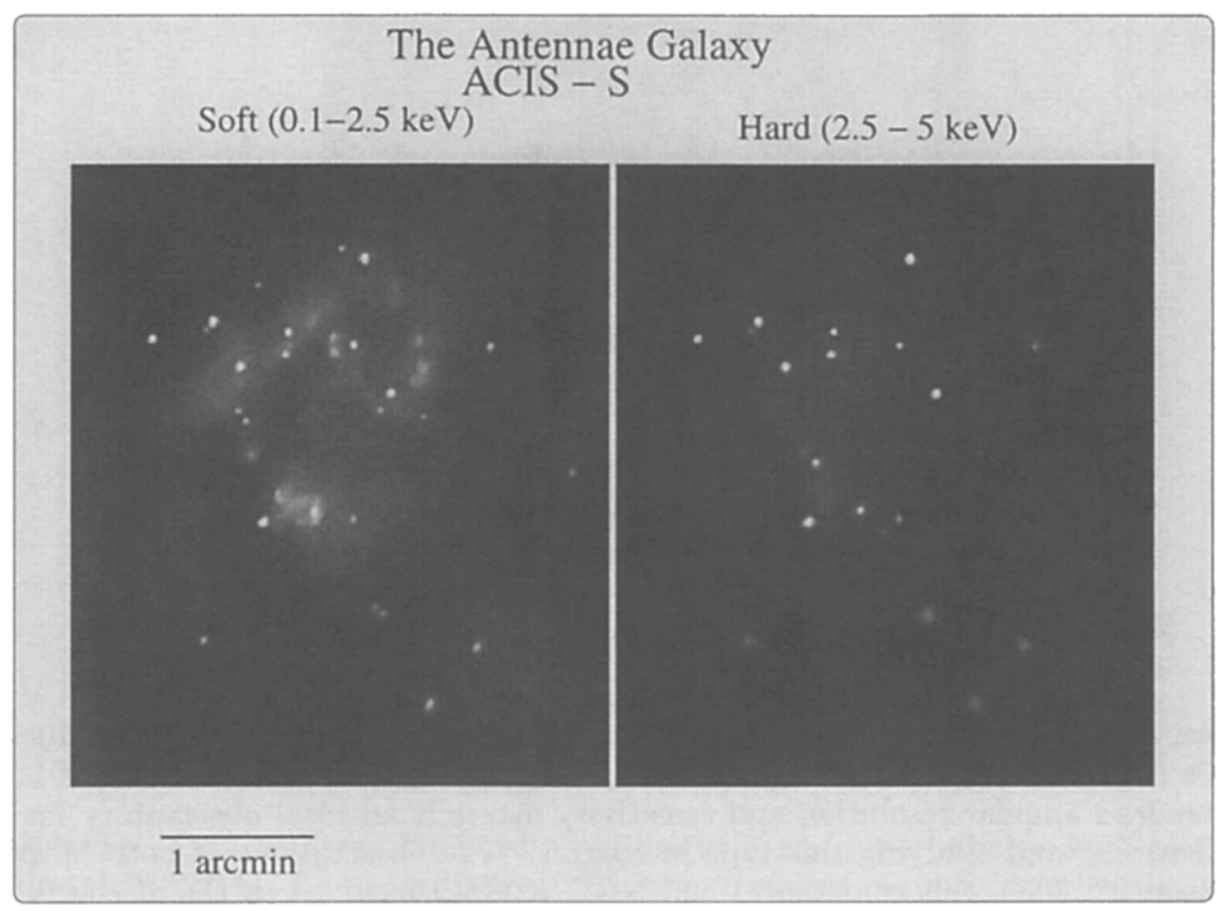

Figure 1. Soft and Hard-band Chandra ACIS images, adaptively smoothed with CIAO 'csmooth'

\section{Chandra Observations of the Antennae}

Fig. 1 shows the Chandra X-ray images of NGC 4038/39 in two energy bands. we detect $\mathrm{X}$-ray emission from the entire interacting system. The emission is complex, with a number of bright point-like sources; diffuse features associated with the star-forming knots; and lower surface brightness emission extending throughout. This emission estends farther South than the stellar disks, suggesting a galaxian outflow or superwind. Fig. 1 shows that most of the diffuse extended emission is soft, while the bright point-like sources have a harder spectrum. Thermal line emission features can be identified in the spectrum of the extended component, pointing to a hot ISM. The total X-ray luminosity of the Antennae galaxies in the Chandra band is $\sim 2 \times 10^{41} \mathrm{ergs} / \mathrm{s}$, with diffuse gaseous and point-like components contributing roughly equal amounts.

\section{Super-Luminous (Super-Eddington) X-ray Sources}

A surprising and interesting result of imaging observations of nearby galaxies has been the number of non-nuclear sources detected with luminosities well above the Eddington luminosity for $1 M_{\odot}$ accreting objects $\left(1.3 \times 10^{38} \mathrm{ergs} / \mathrm{s}\right)$. With the exception of young SNR counterparts, which have been detected in a few instances, and of sources that may have been confused in past low-resolution 


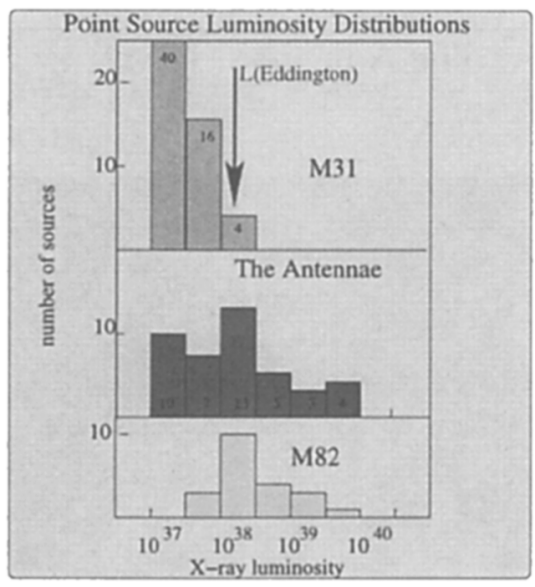

Figure 2. Individual X-ray source luminosity distributions

observations, these sources have been suggested as evidence for massive (10100s $M_{\odot}$ ) black holes binaries (see Fabbiano 1995, 1998, Makishima et al 2000). Chandra's angular resolution and sensitivity makes it an ideal observatory for discovering and studying this type of sources. The observations of both M82 (Prestwich 2000, this conference) and NGC 4038/39 have led to the discovery of a number of point-like super-luminous sources. Fig. 2 shows the histogram of source luminosities in M82 and NGC 4038/39, compared with that of M31 (from Trinchieri \& Fabbiano 1991).

It is evident that the luminosity functions of X-ray sources in these two actively star-forming galaxies are skewed towards higher luminosities, when compared with that of M31, which has an overall older stellar population. This suggests that super-luminous sources have short lifetimes. If these sources are fast-evolving black-hole binaries, and what we see now in star-burst galaxies was the norm at the epoch of galaxy formation, then any spiral galaxy, such as the Milky Way, should have an invisible population of $10-100 \mathrm{~s} M_{\odot}$ black holes, the remnants of spent super-luminous binaries (Fabbiano et al 2000).

\section{The Complex Multi-Phase Interstellar Medium}

Patchy as well as more diffuse soft thermal emission is visible in the Chandra data. The patchy component of the hot ISM is generally correlated with the HII regions and stellar knots in the two interacting galaxy disks. The angular resolution of the Chandra mirrors is such that it is meaningful to pursue direct comparison between Chandra and Hubble data. A comparison between the ISM component of NGC 4038/39 and the warm ISM visible in $H \alpha$ (using archival HST WFPC data), suggests a complex and varied multi-phase ISM. While there is a general resemblance of the spatial distribution of the X-ray and $\mathrm{H \alpha}$ emission, we note both regions where the two surface brightnesses closely follow each others, and regions where hot superbubbles fill-in holes in the $H \alpha$ distribution.

The superbubbles we observe in the ISM of the Antennae galaxies are extraordinary, if we compare them with similar features in the ISM of more normal 


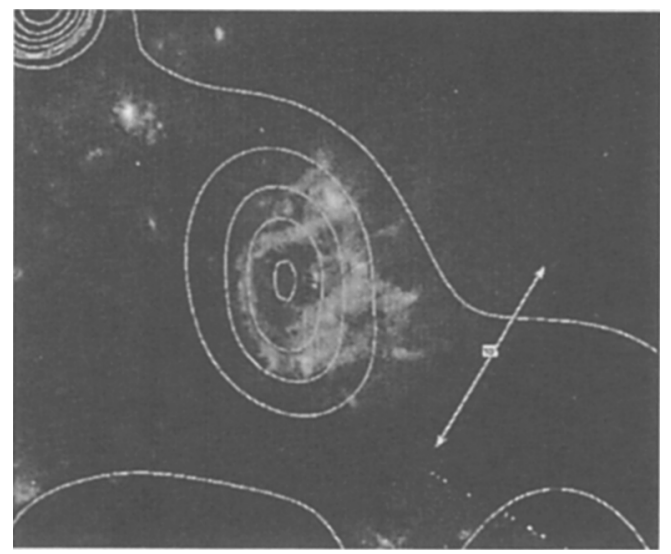

Figure 3. Hot outflowing superbubble: X-ray countours superimposed on the HST WFPC $\mathrm{H} \alpha$ image. The size of the X-ray feature is $\sim 10^{\prime \prime}$ (the 'yardstick' in the figure), corresponding to a $1.4 \mathrm{kpc}$ diameter

galaxies. Typical X-ray luminosities of bright X-ray superbubbles, such as the one shown in fig. 3 , are in the few $\times 10^{39} \mathrm{ergs} / \mathrm{s}$ range, comparable with the entire thermal emission of the nucleus of the nearby starburst galaxy NGC 253 (Fabbiano et al 2000, and refs.therein).

Acknowledgments. This work was supported by NASA contracts NAS839073 (CXC) and NAS8-38248 (HRC).

\section{References}

Fabbiano, G. 1988, Ap. J., 330, 672

Fabbiano, G. 1995a, in 'X-Ray Binaries', W. H. G. Lewin, J. van Paradijs, E. P. J. van den Heuvel, eds., Cambridge Astrophysics Series (Cambridge: University Press), p. 390.

Fabbiano, G. 1998, in 'The Hot Universe', K. Koyama et al, eds., IAU, p. 93.

Fabbiano, G. \& Trinchieri, G. 1983, ApJ, 266, L5

Fabbiano, G., Schweizer, F., \& Mackie, G. 1997, ApJ, 478, 542

Fabbiano, G., Zezas, A. \& Murray, S. S. 2000, ApJ, submitted.

Makishima, K. et al 2000, ApJ535, 632.

Read, A. M., Ponman, T. J., \& Wolstencroft, R. D. 1995, MNRAS, 277, 397

Sansom, A.E., Dotani, T., Okada, K., Yamashita, A., \& Fabbiano, G. 1996, MNRAS, 281, 48

Rubin, V. C., Ford, W. K., \& D'Odorico, S. 1970, ApJ, 160, 801.

Trinchieri, G. \& Fabbiano, G. 1991, ApJ, 382, 82.

Whitmore, B. C., \& Schweizer, F. 1995, AJ, 109, 960 